TITLE:

\title{
Analysis of the effect of core structure upon dineutron correlation using antisymmetrized molecular dynamics
}

\section{AUTHOR(S):}

Kobayashi, Fumiharu; Kanada-En'Yo, Yoshiko

\section{CITATION:}

Kobayashi, Fumiharu ...[et al]. Analysis of the effect of core structure upon dineutron correlation using antisymmetrized molecular dynamics. Physical Review C - Nuclear Physics 2016, 93(2): 024310.

ISSUE DATE:

2016-02-09

URL:

http://hdl.handle.net/2433/218376

RIGHT:

(C) 2016 American Physical Society. 
PHYSICAL REVIEW C 93, 024310 (2016)

\title{
Analysis of the effect of core structure upon dineutron correlation using antisymmetrized molecular dynamics
}

\author{
Fumiharu Kobayashi \\ Department of Physics, Niigata University, Niigata 950-2181, Japan \\ Yoshiko Kanada-En'yo \\ Department of Physics, Kyoto University, Kyoto 606-8502, Japan
}

(Received 28 June 2015; revised manuscript received 16 September 2015; published 9 February 2016)

\begin{abstract}
We extend the method of antisymmetrized molecular dynamics (AMD) to investigate dineutron correlation. We regard the total system as the core plus two valence neutrons in the AMD framework and treat the valence neutron wave functions by multirange Gaussians with the $d$-constraint method, in which the distance between the core and the center of mass of the two neutrons is constrained, to describe the size changing effect and the motion of two neutrons. We apply this method to the ground state of ${ }^{10} \mathrm{Be}$ as an example and investigate the motion of two neutrons around a largely deformed ${ }^{8} \mathrm{Be}$ core by analyzing the two-neutron overlap function around the core. Comparing the results including the different ${ }^{8} \mathrm{Be}$ core structures, we show that the core structure plays an important role in dineutron formation and expansion from the core. The radial fluctuation in the core leads to the expansion of the core potential to the farther region and, as a result, two valence neutrons can be expanded far from the core to form a dineutron. Differently, when the core is less deformed, the dineutron is dissociated by the spin-orbit potential at the surface of the core. We can investigate the dineutron correlation clearly by using the present framework and conclude that the framework is effective for the studies of dineutron correlation.
\end{abstract}

DOI: 10.1103/PhysRevC.93.024310

\section{INTRODUCTION}

Many exotic phenomena have been found in neutron-rich nuclei, and more have been suggested by both theoretical and experimental studies. One such phenomenon is dineutron correlation, a strong spatial correlation between two neutrons coupled to a spin singlet. Although the two neutrons are not bound in a free space, strong dineutron correlation has been theoretically suggested, e.g., in a low-density region of nuclear matter [1,2] or in the neutron-halo or -skin regions of neutron-rich nuclei [3-17]. These studies clarify that the strength of dineutron correlation reflected in the dineutron size changes significantly depending on circumstances such as nuclear density and potential from the core. In addition, the dineutron and diproton correlations have been intensively discussed via $2 n$ and $2 p$ emissions from the unbound nuclei in connection with recent experiments [18-29].

Preceding studies have investigated dineutron correlation in the ground and excited states in certain nuclei, but they have not conducted a systematic investigation, and the formation mechanism underlying dineutron correlation and the dynamics of one or more dineutrons are not well understood. To investigate in detail the dineutron motion with respect to the core, a core $+2 n$ three-body model is useful [3-6,8,9,11]. However, in three-body models, it is somewhat difficult to take various structure changes into account (including excitation, deformation, and clustering), and inert, spherical cores have been assumed in most of the preceding studies. Core excitation and deformation can affect dineutron correlation, and it is necessary to consider changes in the core structure explicitly for a systematic investigation of dineutron correlation.

In our previous studies, using dineutron condensate (DC) wave functions [15-17], we showed that dineutrons in nuclei are fragile and easily broken. To study the properties of dineutron correlation, the following dineutron-breaking effects should be considered. First is the dissociation of a spin-singlet two-neutron pair due to the spin-orbit potential from the core, as discussed in Refs. [17,30,31]. Without the spin-orbit potential, two neutrons tend to be coupled to a spin-singlet pair because of the spin-singlet $s$-wave attraction. However, in reality, two valence neutrons at the nuclear surface feel the spin-orbit potential from the core and tend to occupy the $L S$-favored orbits, resulting in mixing of the spin-triplet pair, i.e., reduction of the spin-singlet dineutron component. We call this effect at the surface due to the spin-orbit potential the " $L S$ dissociation" of a dineutron. Second is the swell in the size of a dineutron in the region far from the core. Two neutrons are not bound in a free space; thus, dineutron correlation vanishes in the asymptotic region far from the core, meaning that the dineutron size becomes infinitely large as the distance from the core increases, as discussed in Ref. [11]. We call such a breaking effect at the region far from the core "dispersion" of a dineutron.

To clarify the properties of the dineutron correlation in neutron-rich nuclei, we use the extended method of antisymmetrized molecular dynamics (AMD) [32-34]. The AMD framework can describe various structures such as deformation and clustering in general nuclei, and it is suitable to describe various core structures. We extend the AMD framework to investigate the degree of dineutron formation at the surface and the degree of expansion of a dineutron tail at the farther region while taking into account the abovementioned breaking effects of a dineutron based on the concept of a core $+2 n$ model. In addition, to analyze the detailed two-neutron motion, we propose a method that enables us to visualize the two-neutron spatial distribution around the core. 
As the first step, we apply the extended AMD framework and the method of analysis to the ground state of ${ }^{10} \mathrm{Be}$, which has a well-deformed ${ }^{8} \mathrm{Be}(2 \alpha)$ core and not so extremely loosely bound two valence neutrons, and discuss the effect of core structure change on the dineutron correlation in this study. We show that the core structure significantly affects the dineutron formation and distribution around the core.

This paper is organized as follows. In Sec. II, we explain the framework used to describe and analyze the dineutron correlation in neutron-rich nuclei. In Sec. III, we apply the framework to the ${ }^{10} \mathrm{Be}$ ground-state system and discuss the dineutron formation and distribution around the ${ }^{8} \mathrm{Be}$ core. We summarize our work in Sec. IV.

\section{FRAMEWORK}

We consider a total $A$-nucleon system composed of an $(A-2)$-nucleon core and two valence neutrons and investigate the two-neutron motion around the core. We describe our framework in this section.

\section{A. AMD wave function}

First, we explain the AMD wave function. An AMD wave function for an $A$-nucleon system is given by the Slater determinant of $A$ single-particle wave functions:

$$
\Phi_{\mathrm{AMD}}(\boldsymbol{Z})=\frac{1}{\sqrt{A !}} \mathcal{A}\left\{\varphi_{1} \cdots \varphi_{A}\right\} .
$$

Here $\mathcal{A}$ is an antisymmetrizer and $\varphi_{i}(i=1, \ldots, A)$ are the single-particle wave functions composed of the Gaussian-type spatial part $\phi_{i}$, the spin part $\chi_{i}$, and the isospin part $\tau_{i}$ as follows:

$$
\begin{aligned}
\varphi_{i} & =\phi_{i} \chi_{i} \tau_{i}, \\
\phi_{i}\left(\boldsymbol{r}_{j}\right) & =\left(\frac{2 v}{\pi}\right)^{3 / 4} \exp \left[-v\left(\boldsymbol{r}_{j}-\boldsymbol{Y}_{i}\right)^{2}\right], \\
\chi_{i} & =\xi_{i \uparrow} \chi_{\uparrow}+\xi_{i \downarrow} \chi_{\downarrow}, \\
\tau_{i} & =p \text { or } n .
\end{aligned}
$$

$\boldsymbol{Z} \equiv\left\{\boldsymbol{Y}_{1}, \ldots, \boldsymbol{Y}_{A}, \boldsymbol{\xi}_{1}, \ldots, \boldsymbol{\xi}_{A}\right\}$ in Eq. (1) are the variational parameters that characterize the Gaussian centers and spin orientations of the $A$ nucleons. $v$ is the Gaussian width characterizing the size of the single-nucleon motion, which is generally common for all nucleons in the standard AMD framework.

In the present AMD framework, the widths can differ from one another ( $v_{i}$ also has the label of the $i$ th single-particle state) and we choose $v_{i}=v_{c}(i \leqslant A-2$; core $)$ and $v_{i}=v_{2 n}$ ( $i \geqslant A-1$; two valence neutrons), as in Ref. [35]. Note that in the case where all the Gaussian widths differ, the centerof-mass motion cannot be separated exactly. Therefore, we treat the center-of-mass motion approximately by reducing the center of mass kinetic energy from the total energy, as described in Sec. III A.

We comment upon the relation between the present AMD framework and the similar fermionic molecular dynamics (FMD) framework [36]. Our AMD framework, in which all the Gaussian widths are not identical, is indeed quite similar to the FMD framework; however, we use phenomenological effective interactions in the Hamiltonian [Eq. (20)], whereas, in a recent FMD calculation, they used effective interactions derived from realistic interactions via the unitary correlation operator method [37].

\section{B. Core + 2n AMD wave function}

We aim to efficiently describe dineutron correlation in neutron-rich nuclei. To this end, we prepare the total wave function in two steps. The $A$-nucleon system is regarded as a core composed of $A-2$ nucleons and two valence neutrons. The two-step treatment of this system is explained below.

First, we prepare the core wave function composed of $A-2$ nucleons:

$$
\Phi_{\text {core }}^{k}=\mathcal{A}\left\{\varphi_{1}^{k}, \ldots, \varphi_{A-2}^{k}\right\},
$$

where $k$ is the label characterizing the core structure, and the core wave function is specified by the parameter sets $\left\{\boldsymbol{Y}_{1}^{k}, \ldots, \boldsymbol{Y}_{A-2}^{k}, \boldsymbol{\xi}_{1}^{k}, \ldots, \boldsymbol{\xi}_{A-2}^{k}\right\}$. We superpose the core wave functions $\left(\sum_{k}\right)$ to consider the core structure change. Here, we consider the ${ }^{8} \mathrm{Be}$ core, as shown later. We use the $2 \alpha$ cluster wave function for the ${ }^{8} \mathrm{Be}$ core for simplicity and choose the $\alpha-\alpha$ distance $d_{\alpha}$ to parametrize the core structure. We can simply generalize the core wave function to the AMD wave function. For example, in principle, it is possible to prepare the deformed cores by performing the $\beta$-constraint variation, and the deformation parameter $\beta$ is chosen as the parameter for the core structure.

After preparing the basis wave functions of the core, we express the $A$-nucleon total wave functions by distributing two neutrons around each core wave function:

$$
\Phi_{\text {total }}^{k}=\frac{1}{\sqrt{A !}} \mathcal{A}\left\{\Phi_{\text {core }}^{k} \varphi_{A-1} \varphi_{A}\right\}
$$

with $\Phi_{\text {total }}^{k}$ being an AMD wave function having the parameters $\left\{\boldsymbol{Y}_{1}^{k}, \ldots, \boldsymbol{Y}_{A-2}^{k}, \boldsymbol{Y}_{A-1}, \boldsymbol{Y}_{A}, \boldsymbol{\xi}_{1}^{k}, \ldots, \boldsymbol{\xi}_{A-2}^{k}, \boldsymbol{\xi}_{A-1}, \boldsymbol{\xi}_{A}\right\}$, where the parameters for the core are fixed. We perform energy variation only on the parameters $\left\{\boldsymbol{Y}_{A-1}, \boldsymbol{Y}_{A}, \boldsymbol{\xi}_{A-1}, \boldsymbol{\xi}_{A}\right\}$ for the two valence neutrons to minimize the total energy under the condition that the mean value of the total center-of-mass position is located at the origin when $v_{2 n}=v_{c} ; \sum_{i} \boldsymbol{Y}_{i}=$ $(0,0,0)$ with $\boldsymbol{Y}_{i}=\boldsymbol{Y}_{i}^{k}-\left(\boldsymbol{Y}_{A-1}+\boldsymbol{Y}_{A}\right) /(A-2)$ for $i \leqslant A-2$. The energy variation for two valence neutrons is performed under the constraint on the distance of the center of mass, $\left(\boldsymbol{Y}_{A-1}+\boldsymbol{Y}_{A}\right) / 2$, of two neutrons from the core, which we call $d_{2 n}$ :

$$
d_{2 n} \equiv\left|\frac{1}{2}\left(\boldsymbol{Y}_{A-1}+\boldsymbol{Y}_{A}\right)-\frac{1}{A-2} \sum_{i \leqslant A-2} \boldsymbol{Y}_{i}\right|,
$$

which depends only on the Gaussian center parameters $\boldsymbol{Y}_{i}$. Performing the $d_{2 n}$-constraint energy variation for $d_{2 n}=d_{2 n, m}$ ( $m=1, \ldots)$, we calculate the two-neutron wave functions near and far from the core. The variations of $\boldsymbol{Y}_{A-1}$ and $\boldsymbol{Y}_{A}$ are performed independently so the two neutrons near the core tend to have the opposite momentum (i.e., the imaginary parts are opposite to one another) and tend to be broken by the $L S$ dissociation effect due to the spin-orbit potential from the core. 
However, two neutrons far from the core tend to have almost the same position, indicating the expansion of a spin-singlet dineutron far from the core owing to the energy variation. A similar constraint is proposed for an $\alpha$ cluster in Ref. [38] and can efficiently describe $\alpha$ cluster development.

For the valence neutrons, we choose different values of the Gaussian width from those of the core nucleons $\left(v_{2 n} \neq v_{c}\right)$. Using the different values of the Gaussian widths $v_{2 n}$, the dineutron dispersion effect is taken into account. Moreover, the description of the spatially expanded tail parts of single-particle wave functions of valence neutrons such as the neutron-halo tail should be improved using multirange Gaussians.

We describe the total system by superposing all wave functions with core deformation $(k)$, core- $2 n$ distance $\left(d_{2 n}\right)$, and $2 n$ size $\left(v_{2 n}\right)$ :

$$
\Phi_{A(J \pi)}=\sum_{K} \sum_{k} \sum_{d_{2 n}} \sum_{\nu_{2 n}} c_{K K} \mathcal{P}_{M K}^{J \pi} \Phi_{\text {total }}^{k}\left(\boldsymbol{Z}, \nu_{2 n}\right) .
$$

$\mathcal{P}_{M K}^{J \pi}$ is the parity and angular momentum projection operator to the eigenstate of $J \pi$. The coefficients $c_{K \kappa}$ ( $\kappa$ is the abbreviation of $\left.\left\{k, d_{2 n}, v_{2 n}\right\}\right)$ are determined by diagonalizing the Hamiltonian [Eq. (20)]. Superposing the wave functions with the set of $\left\{k, d_{2 n}, v_{2 n}\right\}$, we can take the core structure change into account as well as the two-neutron motion near and far from the core while varying their size.

It should be noted that each basis wave function is not a stationary state and has less physical meaning, though it has specific characters in the present AMD framework. For instance, the center of mass of the two valence neutrons is described by the superposition of localized Gaussians with various center positions $d_{2 n}$, but each localized Gaussian is not necessarily a physical state. Also, as for the core state, the core wave function for the physical state is given by the superposition of basis wave functions with different deformations. So, in this paper, we discuss not each basis state but the physical state which is the superposition of many basis states.

\section{C. ${ }^{10}$ Be AMD wave function}

Here, we specify the form of the AMD wave function for ${ }^{10} \mathrm{Be}$. The explicit form of the ${ }^{10} \mathrm{Be}$ wave function is given by

$$
\Phi^{10} \operatorname{Be}(0+)=\sum_{K} \sum_{d_{\alpha}} \sum_{d_{2 n}} \sum_{\nu_{2 n}} c_{K \kappa} \mathcal{P}_{00}^{0+} \Phi_{\text {total }}^{d_{\alpha}}\left(\boldsymbol{Z}, v_{2 n}\right) \text {. }
$$

${ }^{10} \mathrm{Be}$ is regarded as a ${ }^{8} \mathrm{Be}$ core with two valence neutrons.

The ${ }^{8} \mathrm{Be}$ core is described with the $2 \alpha$ cluster wave function for simplicity. An $\alpha$ cluster is composed of four spin- and isospin-saturated nucleons in $(0 s)^{4}$ with the same Gaussian center and width. We choose $\alpha-\alpha$ distance $d_{\alpha}$ as the parameter characterizing the core structure $\left[k=d_{\alpha}\right.$ in Eq. (9)]. We prepare three types of cores with $d_{\alpha}=2,3,4 \mathrm{fm}$ to describe the core structure fluctuation. The superposition of these values gives the good convergence in the ${ }^{10} \mathrm{Be}$ ground state. The Gaussian widths of the core nucleons are fixed to $v_{c}=0.235 \mathrm{fm}^{-2}$, used in the preceding works on ${ }^{10} \mathrm{Be}[39,40]$.

Then, we distribute two neutrons around each core with $d_{\alpha}=2,3,4 \mathrm{fm}$ under the $d_{2 n}$ constraint [Eq. (8)]. Here, we choose $d_{2 n}$ to be $d_{2 n}=d_{\min } d^{m-1}\left(m=1, \ldots, m_{\max }\right)$. We define $d_{\min } d^{m_{\max }-1}=d_{\max }$. We set $\left(m_{\max }, d_{\min }, d_{\max }\right)=(8,1,5)$. We verified that the ${ }^{10} \mathrm{Be}$ ground-state energy is lowered by only $100 \mathrm{keV}$ at most if the number of bases or the maximum value of $d_{\max }$ is increased. When $d_{2 n}$ is small and two neutrons are distributed near the core, the $L S$ dissociation for a dineutron plays an important role and the two neutrons mainly occupy the lowest allowed orbit $0 p_{3 / 2}$ to gain the spin-orbit potential from the core. However, when $d_{2 n}$ is large, the tail of the dineutron center-of-mass motion far from the core can be well described. The present parameter set $d_{\alpha}=2,3,4 \mathrm{fm}$ and $\left(m_{\max }, d_{\min }, d_{\max }\right)=(8,1,5)$ are sufficient to describe the ground state where $2 \alpha$ s in the core, and the core and the valence neutrons are not extremely loosely bound. Note that a larger value for $d_{\max }$ should be adopted to describe more loosely bound neutron-rich nuclei. Also for $d_{\alpha}$, a larger value for the maximum value should be used for cluster excited states near the $\alpha$-decay threshold, because such states are expected to have a long tail of the $\alpha$-cluster probability in the outer region.

In each $d_{2 n}$-constraint variation, we fix the Gaussian widths of the two valence neutrons to be $v_{2 n}=0.235,0.125$, or $0.08 \mathrm{fm}^{-2}$ and perform variation for each $\nu_{2 n}$ value. The superposition of three types of bases with different $v_{2 n}$ values for each $d_{2 n}$ can describe the dineutron size change.

At the nuclear surface, the $L S$ dissociation of a dineutron is predominant and dineutron correlation is suppressed. To more effectively describe the dineutron formation at the surface, we additionally superpose the bases prepared with the $d_{2 n}$-constraint variation without the spin-orbit force $\left(v_{L S}=0\right.$ $\mathrm{MeV}$ ) when $d_{2 n}$ is small. Here, we prepare the bases without the spin-orbit force when $d_{2 n}<2 \mathrm{fm}$ (four bases) for each $\left(d_{\alpha}, v_{2 n}\right)$ set.

We summarize the number of bases used to describe ${ }^{10} \mathrm{Be}$. We use three types of ${ }^{8} \mathrm{Be}$ cores $\left(d_{\alpha}=2,3,4 \mathrm{fm}\right)$ and three widths of the two valence neutrons for each core $\left(v_{2 n}=0.235\right.$, $\left.0.125,0.08 \mathrm{fm}^{-2}\right)$. We perform the energy variation on the two valence neutrons in each $\left(d_{\alpha}, v_{2 n}\right)$ set under the $d_{2 n}$ constraint. We choose eight values for $d_{2 n}$ with $v_{L S}=1600 \mathrm{MeV}$ (this value is explained later) and four values for $d_{2 n}(<2 \mathrm{fm})$ with $v_{L S}=0 \mathrm{MeV}$. Then, the number of bases used to describe ${ }^{10} \mathrm{Be}$ in the present full calculation is $(8+4) \times 3 \times 3=108$.

In this study, we would like to examine the effect of core structure on dineutron correlation in ${ }^{10} \mathrm{Be}$. To this end, we compare the results obtained by two types of calculations. One is the full calculation mentioned above where the bases with $d_{\alpha}=2,3,4 \mathrm{fm}$ are superposed to describe the fluctuation of the $\alpha-\alpha$ distance in the core structure, i.e., the shape fluctuation of the core. The other is the truncated calculation where only the bases with $d_{\alpha}=2 \mathrm{fm}$ are superposed, which gives the state with the smaller core deformation. We denote this calculation as the "fixed- $d_{\alpha}$ " calculation. Note that, strictly speaking, the fixed core in the fixed- $d_{\alpha}$ calculation is not a physical state for the core as mentioned previously. However, we introduce the fixed core as a reference state for comparison with the full calculation. The bases in the fixed- $d_{\alpha}$ calculation are recalculated because we use the interaction parameters modified from those of the full calculation to reproduce the two-neutron separation energy in each calculation. The main difference between the full and fixed- $d_{\alpha}$ calculations is whether 
or not the component of the larger core shape is taken into account. In Sec. III, we mainly focus on the difference in the dineutron correlation coming from this component.

\section{D. $2 n$ overlap function}

For analysis to examine the dineutron correlation around the core, we propose a method using a $2 n$ overlap function. The $2 n$ overlap function $f$ is defined as

$$
\begin{aligned}
f\left(\boldsymbol{r}, \boldsymbol{r}_{G}\right) \equiv & \mathcal{A}\left\langle\Phi_{\text {core }}^{d_{\alpha}} \delta\left(\boldsymbol{r}_{A-1}-\boldsymbol{r}_{n_{1}}\right) \chi_{\uparrow} \tau_{n}\right. \\
& \left.\times \delta\left(\boldsymbol{r}_{A}-\boldsymbol{r}_{n_{2}}\right) \chi_{\downarrow} \tau_{n} \mid \Phi_{10} \operatorname{Be}(0+)\right), \\
\boldsymbol{r}= & \boldsymbol{r}_{n_{2}}-\boldsymbol{r}_{n_{1}}, \quad \boldsymbol{r}_{G}=\left(\boldsymbol{r}_{n_{1}}+\boldsymbol{r}_{n_{2}}\right) / 2,
\end{aligned}
$$

where $\Phi_{\text {core }}^{d_{\alpha}}$ is the ${ }^{8} \mathrm{Be}$ core wave function [Eq. (6)] specified by the parameter $d_{\alpha}$. We use the core with $d_{\alpha}=3 \mathrm{fm}$ in the full calculation and that with $d_{\alpha}=2 \mathrm{fm}$ in the fixed- $d_{\alpha}$ calculation, which gives the largest overlap with the ground state obtained in each calculation. $f\left(\boldsymbol{r}, \boldsymbol{r}_{G}\right)$ is defined as a function of the relative and center-of-mass coordinates of two neutrons defined by the two-neutron coordinates, $\boldsymbol{r}_{n_{1}}$ and $\boldsymbol{r}_{n_{2}}$, as shown in Eq. (12). We omit the recoil of the core and locate its center of mass in the bra and ket states at the origin to measure the largest overlap between these states. The quantity $f\left(\boldsymbol{r}, \boldsymbol{r}_{G}\right)$ brings out information about the spatial distribution of spin-up and -down neutrons in $\Phi^{10} \mathrm{Be}$ as a function of $\boldsymbol{r}$ and $\boldsymbol{r}_{G}$. This quantity corresponds to a type of reduced width amplitude extended to the three-body case. Although the definition of the two-body density is nontrivial, we define $f\left(\boldsymbol{r}, \boldsymbol{r}_{G}\right)$ to be totally antisymmetrized, and $f\left(\boldsymbol{r}, \boldsymbol{r}_{G}\right)$ directly reflects the information about the spatial distribution of the two neutrons. The $2 n$ overlap function is useful for analysis of the dineutron correlation, as accomplished using the three-body model.

In particular, we are interested in the component of the dineutron where two neutrons are coupled to a spin singlet with a relative $s$ wave $(l=0)$. We focus on the dineutron in the $S$ wave $(L=0)$ with respect to the core for simplicity. Here, we use the label "s" $(l=0)$ for the angular momentum for the relative coordinate $\boldsymbol{r}$ and "S" $(L=0)$ for the angular momentum for the center-of-mass coordinate $\boldsymbol{r}_{G}$. Thus, we project the angular momenta of the relative and center-of-mass motions of the two neutrons to the $s$ and $S$ waves, respectively, and consider the $2 n$ overlap function, $f_{l=L=0}^{S=0}\left(r, r_{G}\right)$, for the states projected to $l=L=0$ as a function of $r=|\boldsymbol{r}|$ and $r_{G}=\left|\boldsymbol{r}_{G}\right|$. The details of the calculation of $f_{l=L=0}^{S=0}$ are given in the Appendix.

Using the $2 n$ overlap function $f_{l=L=0}^{S=0}$, we calculate the probability

$$
P_{l=L=0}^{S=0} \equiv \int r^{2} d r r_{G}^{2} d r_{G}\left|f_{l=L=0}^{S=0}\left(r, r_{G}\right)\right|^{2},
$$

which corresponds to the component of the spin-singlet and relative $s$-wave $2 n$ pair moving in the $S$ wave around the core.

We also calculate the root-mean-square distance for $r$ and $r_{G}$ of the two neutrons in the $S=0$ and $l=L=0$ components:

$$
\begin{aligned}
& \sqrt{\left\langle r^{2}\right\rangle_{l=L=0}^{S=0}} \\
& =\left(\int r^{2} d r r_{G}^{2} d r_{G} r^{2}\left|f_{l=L=0}^{S=0}\left(r, r_{G}\right)\right|^{2} / P_{l=L=0}^{S=0}\right)^{1 / 2}, \\
& \sqrt{\left\langle r_{G}^{2}\right\rangle_{l=L=0}^{S=0}} \\
& =\left(\int r^{2} d r r_{G}^{2} d r_{G} r_{G}^{2}\left|f_{l=L=0}^{S=0}\left(r, r_{G}\right)\right|^{2} / P_{l=L=0}^{S=0}\right)^{1 / 2},
\end{aligned}
$$

which correspond to the dineutron size and the expansion from the core, respectively.

Note that the asymmetry with respect to the exchange, $r / 2 \leftrightarrow r_{G}$ in $f_{l=L=0}^{S=0}\left(r, r_{G}\right)$, reflects the mixing of singleparticle orbits for two neutrons of different parity in the $S=0$ and $l=L=0$ components. We decompose $f_{l=L=0}^{S=0}$ and $P_{l=L=0}^{S=0}$ into symmetric and antisymmetric components as follows:

$$
\begin{aligned}
f_{l=L=0}^{S=0}\left(r, r_{G}\right) & =f_{l=L=0}^{S=0(++)}\left(r, r_{G}\right)+f_{l=L=0}^{S=0(--)}\left(r, r_{G}\right), \\
P_{l=L=0}^{S=0} & =P_{l=L=0}^{S=0(++)}+P_{l=L=0}^{S=0(--)},
\end{aligned}
$$

where $f_{l=L=0}^{S=0( \pm \pm)}$ and $P_{l=L=0}^{S=0( \pm \pm)}$ are defined as

$$
\begin{aligned}
f_{l=L=0}^{S=0( \pm \pm)}\left(r, r_{G}\right) & \equiv \frac{1 \pm \mathcal{P}_{r / 2 \leftrightarrow r_{G}}}{2} f_{l=L=0}^{S=0}\left(r, r_{G}\right) \\
& =\frac{1}{2}\left(f_{l=L=0}^{S=0}\left(r, r_{G}\right) \pm f_{l=L=0}^{S=0}\left(2 r_{G}, r / 2\right)\right), \\
P_{l=L=0}^{S=0( \pm \pm)} & \equiv \int r^{2} d r r_{G}^{2} d r_{G}\left|f_{l=L=0}^{S=0( \pm \pm)}\left(r, r_{G}\right)\right|^{2} .
\end{aligned}
$$

Here $\mathcal{P}_{r / 2 \leftrightarrow r_{G}}$ is the $r / 2 \leftrightarrow r_{G}$ exchange operator and the projection $\left(1 \pm \mathcal{P}_{r / 2 \leftrightarrow r_{G}}\right) / 2$ is equivalent to the double projection $\left(1 \pm \mathcal{P}_{\boldsymbol{r}_{n 1}}\right) / 2 \times\left(1 \pm \mathcal{P}_{\boldsymbol{r}_{n 2}}\right) / 2$ of the single-particle parities on $f_{l=L=0}^{S=0}\left(r / 2, r_{G}\right)$, as shown in the Appendix. $\mathcal{P}_{\boldsymbol{r}_{n 1,2}}$ are the space reflection operators of $\boldsymbol{r}_{n_{1,2}} \rightarrow-\boldsymbol{r}_{n_{1,2}}$. This means that $f_{l=L=0}^{S=0(++)}\left(f_{l=L=0}^{S=0(--)}\right)$ and $P_{l=L=0}^{S=0(++)}\left(P_{l=L=0}^{S=0(--)}\right)$ indicate the contributions of pure positive-parity (negative-parity) singleparticle states of two neutrons in $f_{l=L=0}^{S=0}$ and $P_{l=L=0}^{S=0}$. We hereafter label $f_{l=L=0}^{S=0( \pm \pm)}$ as the (++) or (- ) component of the $2 n$ overlap function. The dineutron correlation in ${ }^{10} \mathrm{Be}$ is seen in $f_{l=L=0}^{S=0}$ as the coherent mixing of the minor $f_{l=L=0}^{S=0(++)}$ into the major $f_{l=L=0}^{S=0(--)}$, as shown in Sec. III C.

\section{RESULT}

\section{A. Effective Hamiltonian}

In the present work, we use the Hamiltonian

$$
H=T-T_{G}+V_{\text {cent }}+V_{\mathrm{LS}}+V_{\text {Coul }},
$$

where $T$ and $T_{G}$ are the total and center-of-mass kinetic energies. In the present framework, since all the Gaussian widths in the present AMD wave functions are not equal, the center-of-mass motion cannot be removed exactly. We therefore treat the center-of-mass motion approximately by reducing the expectation value of $T_{G}$ from the total Hamiltonian. $V_{\text {Coul }}$ is the Coulomb force that is approximated by the summation of seven Gaussians. $V_{\text {cent }}$ and $V_{\mathrm{LS}}$ are the 
effective central and spin-orbit interactions. We use the Volkov no. 2 force [41] as $V_{\text {cent }}$ and the spin-orbit part of the G3RS force [42] as $V_{\mathrm{LS}}$. In this work, we choose the strength of the spin-orbit force to be $v_{\mathrm{LS}}=1600 \mathrm{MeV}$, as has been used in the previous works on the subject of ${ }^{10} \mathrm{Be}[39,40]$. The Bartlett and Heisenberg parameters in the central force are $b=h=0.125$ which reproduce the deuteron binding energy and the $n-n$ unbound feature. We use the Majorana parameter $m=0.60$ as it was used in Refs. $[39,40]$ in the full calculation. In the fixed- $d_{\alpha}$ calculation, we choose $m=0.64$ to give almost the same two-neutron separation energy, $S_{2 n}$, that was obtained in the full calculation.

\section{B. ${ }^{10} \mathrm{Be}$ fundamental properties}

We calculate the two-neutron separation energy, $S_{2 n}$, the matter, proton, and neutron radii, $r_{m, p, n}$, and the expectation value of the squared neutron total spin, $\left\langle S_{n}^{2}\right\rangle$, shown in Table I. The experimental proton radius is calculated from the measured charge radius by using the equation in Ref. [44],

$$
r_{p}^{2}=r_{c}^{2}-R_{p}^{2}-\frac{N}{Z} R_{n}^{2}-\frac{3 \hbar^{2}}{4 m_{p}^{2} c^{2}},
$$

where $r_{c}, R_{p}$, and $R_{n}$ are the charge radii of a nucleus, a proton, and a neutron, respectively. The proton and neutron charge radii are 0.8791 and $-0.1149 \mathrm{fm}$ referred from Ref. [45]. The last term is the Darwin-Foldy term, where $m_{p}$ is the proton mass [46]. The two-neutron separation energy is calculated as the difference between the total binding energy and the core binding energy,

$$
S_{2 n}=-\left(\left\langle\Phi_{{ }^{10} \mathrm{Be}(0+)}|H| \Phi_{{ }^{10} \mathrm{Be}(0+)}\right\rangle-\left\langle\Phi_{8^{\mathrm{Be}(0+)}}|H| \Phi_{8^{\mathrm{Be}(0+)}}\right\rangle\right),
$$

where $\Phi_{8} \mathrm{Be}(0+)$ is the superposition of the ${ }^{8} \mathrm{Be}$ core wave functions with $d_{\alpha}=2,3,4 \mathrm{fm}$ in the full calculation and that with $d_{\alpha}=2 \mathrm{fm}$ in the fixed- $d_{\alpha}$ calculation, and they are projected onto $J^{\pi}=0^{+}$. We show the binding energies of the ${ }^{8} \mathrm{Be}$ cores and the ${ }^{10} \mathrm{Be}$ bases giving the lowest energies with $d_{\alpha}=2,3$, and 4 fm projected onto $J^{\pi}=0^{+}$calculated with $m=0.60$ in Table II. Although $d_{\alpha}=3 \mathrm{fm}$ gives the lowest energy in ${ }^{10} \mathrm{Be}$, we choose the $d_{\alpha}=2$ bases in the fixed- $d_{\alpha}$ calculation to compare the less deformed case with the superposing calculation. We superpose these bases of ${ }^{10} \mathrm{Be}$

TABLE I. The used Majorana parameter $m$, the two-neutron separation energy $S_{2 n}$, the root-mean-square radii $r_{m, p, n}$ of matter, protons, and neutrons, and the expectation value of the squared neutron total $\operatorname{spin}\left\langle S_{n}^{2}\right\rangle$ in the full and fixed- $d_{\alpha}$ (fixed) calculations. The experimental values of the matter radii are referred from Ref. [43] and the proton radius is calculated from the experimental charge radius in Ref. [44].

\begin{tabular}{lccllll}
\hline \hline & $\begin{array}{c}m \\
(\mathrm{MeV})\end{array}$ & $\begin{array}{c}S_{2 n} \\
(\mathrm{fm})\end{array}$ & $\begin{array}{c}r_{m} \\
(\mathrm{fm})\end{array}$ & $\begin{array}{c}r_{p} \\
(\mathrm{fm})\end{array}$ & $r_{n}$ & $\left\langle S_{n}^{2}\right\rangle$ \\
\hline Full & 0.60 & 6.71 & 2.43 & 2.22 & 2.51 & 0.39 \\
Fixed & 0.64 & 6.34 & 2.33 & 2.01 & 2.38 & 0.50 \\
Expt. & & 8.48 & $2.30(2)$ & $2.18(2)$ & & \\
\hline \hline
\end{tabular}

TABLE II. The binding energies of the ${ }^{8} \mathrm{Be}$ cores and the lowest binding energies of the ${ }^{10} \mathrm{Be}$ bases with $d_{\alpha}=2,3$, and $4 \mathrm{fm}$ (in MeV). We use the parameter $m=0.60$ in the central force.

\begin{tabular}{lccc}
\hline \hline & $d_{\alpha}=2$ & $d_{\alpha}=3$ & $d_{\alpha}=4$ \\
\hline${ }^{8} \mathrm{Be}$ & 46.33 & 51.49 & 52.65 \\
${ }^{10} \mathrm{Be}$ & 54.26 & 55.80 & 51.26 \\
\hline \hline
\end{tabular}

with $d_{\alpha}=2,3,4 \mathrm{fm}$ to get the binding energy of $60.42 \mathrm{MeV}$ (full calculation). For the calculation with the fixed value of $d_{\alpha}=2 \mathrm{fm}$ (fixed- $d_{\alpha}$ calculation), we use the modified interaction of $m=0.64$ to fit the two-neutron separation energy of the full calculation as mentioned previously, and get the binding energy of $49.63 \mathrm{MeV}$. The root-mean-square radii of matter, protons, and neutrons are larger in the full calculation than those in the fixed- $d_{\alpha}$ calculation. This is natural because the core size becomes larger in the full calculation due to the fluctuation in the $\alpha-\alpha$ distance.

We show the neutron spin expectation value, $\left\langle S_{n}^{2}\right\rangle$, in Table I. In the present calculation, the ${ }^{8} \mathrm{Be}$ core has zero proton and neutron spins and, therefore, $\left\langle S_{n}^{2}\right\rangle$ indicates the squared spin expectation value of the two valence neutrons. The finite value of $\left\langle S_{n}^{2}\right\rangle$ reflects the spin-triplet component, that is, the degree of the $L S$ dissociation of a dineutron. $\left\langle S_{n}^{2}\right\rangle$ is larger in the fixed- $d_{\alpha}$ calculation than that in the full calculation, indicating that the $L S$ dissociation increases when the core structure is fixed to be small. This point is discussed later in connection with the dineutron enhancement due to the core structure change.

We checked the relationship between the present AMD wave function and the DC wave function used in our previous work [40]. For the DC wave function, we assume a spinsinglet $2 n$ pair around a core, and we superposed the DC wave functions with the AMD wave functions. The main role of the DC wave function discussed in Ref. [40] is to describe the dineutron-tail component in ${ }^{10} \mathrm{Be}$. If we superpose the DC wave functions used in Ref. [40] with the present AMD wave functions, the ground-state energy is lowered by only $300 \mathrm{keV}$ at most and the other properties are largely unchanged. This means that the contribution of the DC wave function is minor, and that the structure as well as the dineutron tail can be well described with the present $d_{2 n}$-constrained AMD wave functions, at least for a ${ }^{10} \mathrm{Be}$ system with sufficiently bound valence neutrons.

\section{Effect of core structure change on dineutron correlation in ${ }^{10} \mathrm{Be}$}

In this section, we discuss the dineutron correlation in the ground state of ${ }^{10} \mathrm{Be}$, focusing mainly on the effect of ${ }^{8} \mathrm{Be}$ core structure change.

As an example of the analysis of dineutron correlation using the $2 n$ overlap function, we plot the $2 n$ overlap functions, $f_{l=L=0}^{S=0}$ and $f_{l=L=0}^{S=0( \pm \pm)}$, in the full calculation as functions of $\left(r / 2, r_{G}\right)$ in Fig. 1. To show the asymmetry with respect to the $r_{G}=r / 2$ line, we show the $r / 2-r_{G}$ plot instead of the $r-r_{G}$ plot. There are two peaks in $f_{l=L=0}^{S=0}$ and $f_{l=L=0}^{S=0(--)}$ and three peaks in $f_{l=L=0}^{S=0(++)}$. Hereafter, we refer to the peak in 


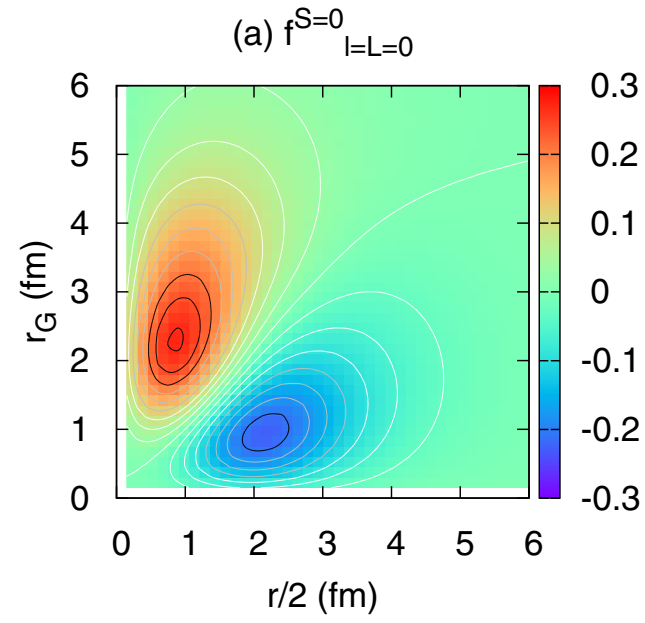

(b) $f^{S=0(--)}{ }_{l=L}=0$
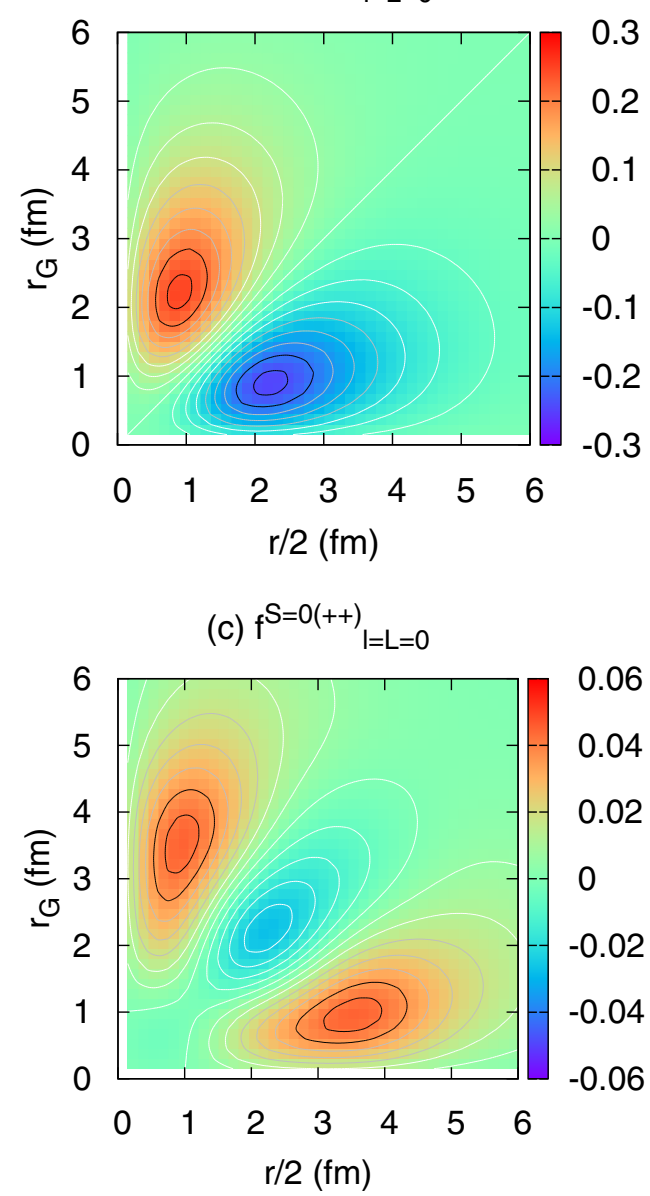

FIG. 1. The $2 n$ overlap functions of (a) $f_{l=L=0}^{S=0}$, (b) $f_{l=L=0}^{S=0(--)}$, and (c) $f_{l=L=0}^{S=0(++)}$ in the full calculation as functions of $\left(r / 2, r_{G}\right)$. For the guide, the colors of the lines corresponding to the region where the absolute amplitude is largest, intermediate, and smallest are black, gray, and white, respectively.

the $r_{G}>r / 2$ region of $f_{l=L=0}^{S=0}$ as the dineutron peak and the peak in the $r_{G}<r / 2$ region of $f_{l=L=0}^{S=0}$ as the cigar peak. The two-peak structure in $f_{l=L=0}^{S=0(--)}$ comes from the two neutrons occupying the $p^{2}$ orbits and the three-peak structure in $f_{l=L=0}^{S=0(++)}$ comes from those occupying the $(s d)^{2}$ orbits. The (--) and (++) components of the $2 n$ overlap function, $f_{l=L=0}^{S=0(-)}$ and $f_{l=L=0}^{S=0(++)}$, are antisymmetric and symmetric with respect to the $r_{G}=r / 2$ line, respectively. In general, without the mixing of different parity contributions for the single-particle orbits, the absolute amplitudes of the dineutron and cigar peaks are exactly the same, and we do not describe the case as dineutron correlation. In other words, dineutron correlation is reflected in the asymmetry between the dineutron and cigar peaks with respect to the $r_{G}=r / 2$ line in $f_{l=L=0}^{S=0}$. In the present case, the asymmetry in $f_{l=L=0}^{S=0}$ comes from the mixing of the minor $(s d)^{2}$ component $\left(f_{l=L=0}^{S=0(++)}\right)$ into the major $p^{2}$ component $\left(f_{l=L=0}^{S=0(--)}\right)$, as shown in Eq. (16). The relative phases at the dineutron peaks $(r / 2 \sim 1 \mathrm{fm})$ in $f_{l=L=0}^{S=0(--)}$ and $f_{l=L=0}^{S=0(++)}$ are coherent; however, those at the cigar peaks $\left(r_{G} \sim 1 \mathrm{fm}\right)$ in $f_{l=L=0}^{S=0(-)}$ and $f_{l=L=0}^{S=0(++)}$ are incoherent, leading to asymmetry between the dineutron and cigar peaks. The dineutron enhancement is seen in the obvious asymmetry between the dineutron and cigar components coming from significant mixing of the different-parity single-particle orbits for each valence neutron.

We now investigate the dependence of the degree of dineutron enhancement on the core structure by comparing the results obtained from the full and fixed- $d_{\alpha}$ calculations. In Table III, we show some properties of the $2 n$ pair in two calculations. As mentioned above, the mixing of the differentparity single-particle orbits for the two neutrons reflects the dineutron correlation; in other words, the mixing ratio of $P_{l=L=0}^{S=0(++)}$ into $P_{l=L=0}^{S=0(--)}$ reflects the strength of dineutron correlation. The mixing ratio $P_{l=L=0}^{S=0(++)} / P_{l=L=0}^{S=0(--)}$ is $5.1 \%$ in the full calculation and $3.0 \%$ in the fixed $-d_{\alpha}$ calculation, meaning that the large deformation and large shape fluctuation in the ${ }^{8} \mathrm{Be}$ core structure enhance the dineutron correlation. The stronger dineutron correlation in the full calculation is also reflected in the slightly smaller value of $\sqrt{\left\langle r^{2}\right\rangle_{l=L=0}^{S=0}}$ than that in the fixed- $d_{\alpha}$ calculation.

The reason for which the core structure change enhances the dineutron correlation is as follows: when the core deformation is fixed to be small in the fixed- $d_{\alpha}$ calculation, two valence neutrons are distributed near the core to a larger extent and they feel the stronger spin-orbit potential at the surface. In the ${ }^{10} \mathrm{Be}$

TABLE III. The probabilities of the spin-singlet $2 n$ pair $P_{l=L=0}^{S=0}$, those of the positive- or negative-parity components $P_{l=L=0}^{S=0( \pm \pm)}$, the mixing ratio of the positive-parity component to the negative-parity component $P_{l=L=0}^{S=0(++)} / P_{l=L=0}^{S=0(-)}$ (tagged as "ratio"), and the rootmean-square expectation values of the relative and center-of-mass distances of the $2 n$ pair, $\sqrt{\left\langle r^{2}\right\rangle_{l=L=0}^{S=0}}$ and $\sqrt{\left\langle r_{G}^{2}\right\rangle_{l=L=0}^{S=0}}$, in the full and fixed- $d_{\alpha}$ (fixed) calculations.

\begin{tabular}{lcccccc}
\hline \hline & $P_{l=L=0}^{S=0}$ & $P_{l=L=0}^{S=0(-)}$ & $P_{l=L=0}^{S=0(++)}$ & $\begin{array}{r}\text { ratio } \\
(\%)\end{array}$ & $\begin{array}{c}\sqrt{\left\langle r^{2}\right\rangle_{l=L=0}^{S=0}} \\
(\mathrm{fm})\end{array}$ & $\begin{array}{c}\sqrt{\left\langle r_{G}^{2}\right\rangle_{l=L=0}^{S=0}} \\
(\mathrm{fm})\end{array}$ \\
\hline Full & 0.515 & 0.491 & 0.025 & 5.1 & 3.70 & 2.53 \\
Fixed & 0.546 & 0.530 & 0.016 & 3.0 & 3.75 & 2.37 \\
\hline \hline
\end{tabular}


case, the $L S$-favored orbit of $0 p_{3 / 2}$ is partially unoccupied and the valence neutrons are favored energetically to occupy the $0 p_{3 / 2}$ orbit. Under the smaller core deformation, the simplest shell-model component (two neutrons occupy only the lowest shell) is predominant because of the spin-orbit potential from the core and, therefore, dineutron correlation is not greatly enhanced because of the $L S$ dissociation effect. However, when the core deformation becomes larger, the mean field generated by the core expands to the farther region so that two valence neutrons can be radially expanded from the core to form a spin-singlet compact dineutron. As a result, $\sqrt{\left\langle r_{G}^{2}\right\rangle_{l=L=0}^{S=0}}$ is larger in the full calculation. Moreover, the spin-orbit potential becomes weaker in the region far from the core, resulting in the suppression of the $L S$ dissociation effect on the dineutron. The suppression of the $L S$ dissociation in the full calculation is seen by the smaller spin-triplet component $\left(\left\langle S_{n}^{2}\right\rangle / 2\right)$ than that in the fixed- $d_{\alpha}$ calculation, as already shown in Table I.

However, it should be noted that the $P_{l=L=0}^{S=0}$ value itself is smaller in the full calculation than that in the fixed$d_{\alpha}$ calculation. This is due to the fact that, if the core deformation becomes larger, the spin-singlet $2 n$ components in the strong-coupling channels between the core and $2 n$ [e.g., $\left(L_{\text {core }}=2\right) \otimes(L=2)=0$ which is projected out in the calculations of $P_{l=L=0}^{S=0}$ and $\left.P_{l=L=0}^{S=0( \pm \pm)}\right]$ are mixed to a greater extent. We emphasize that the mixing of the $(++)$ component of the single-particle orbits of $2 n$ into the dominant $(--)$ component increases, reflecting the enhancement of the dineutron correlation.

To investigate the effect of the core structure on the $2 n$ properties in more detail, we compare the $2 n$ overlap functions in the full and fixed- $d_{\alpha}$ calculations. In Fig. 2, we plot the absolute values of the $2 n$ overlap function, $\left|f_{l=L=0}^{S=0}\right|$, projected onto the $r / 2$ axis obtained in the full and fixed- $d_{\alpha}$ calculations. It can be seen that the dineutron peak $(r / 2 \sim 1 \mathrm{fm})$ is larger than the cigar peak $(r / 2 \sim 2 \mathrm{fm})$ in both calculations. However, the difference between the dineutron and cigar peaks is larger

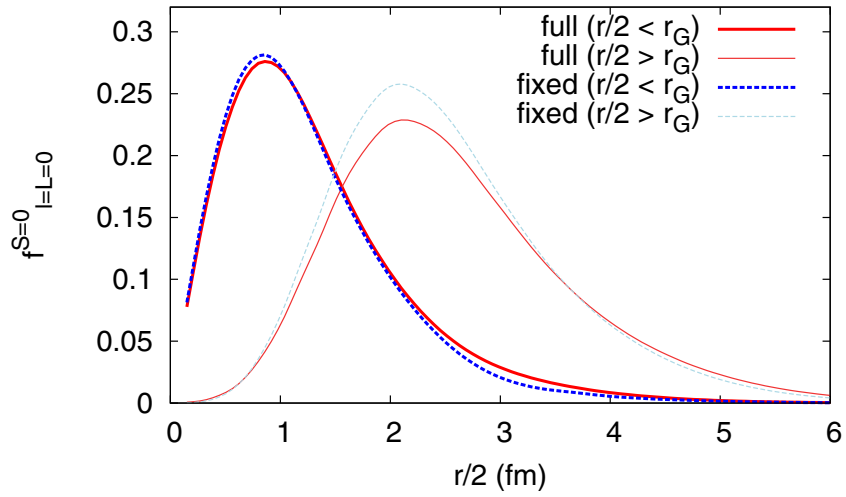

FIG. 2. The absolute values of the projection of the $2 n$ overlap functions of $f_{l=L=0}^{S=0}$ onto the $r / 2$ axis in the full and fixed- $d_{\alpha}$ (fixed) calculations. The red solid lines correspond to the full calculation and the blue dashed lines correspond to the fixed $-d_{\alpha}$ calculation. The thick lines are the dineutron component (the amplitudes in the $r / 2<r_{G}$ region) and the thin lines are the cigar component (the amplitudes in the $r / 2>r_{G}$ region). in the full calculation than that in the fixed- $d_{\alpha}$ calculation. This means that the dineutron correlation is enhanced due to the fluctuation in the $\alpha-\alpha$ distance, as discussed in connection with the mixing of $P_{l=L=0}^{S=0(++)}$ into $P_{l=L=0}^{S=0(-)}$. It should be noticed that the absolute amplitudes of both the dineutron and cigar peaks themselves become smaller in the full calculation because of the mixing of the $\left(L_{\text {core }} \neq 0\right) \otimes(L \neq 0)=0$ components, as mentioned above. Introducing the fluctuation in the distance between $2 \alpha \mathrm{s}$, the cigar peak decreases more than the dineutron peak and the difference between these peaks is certainly increased, indicating dineutron enhancement depending on the core structure change.

As mentioned above, the difference in the results in the full and fixed calculations mainly comes from the mixing of the component of the more largely deformed core. Therefore, for instance, the comparison between the full calculation and a calculation where $d_{\alpha}$ is fixed to $4 \mathrm{fm}$ gives smaller differences than that shown in the present result.

\section{Utility of the $d_{2 n}$-constraint calculation}

Finally, to show the utility of the present $d_{2 n}$-constraint calculation, we compare the dineutron-tail component obtained by the full calculation with that obtained by the single basis having the parameters $\left(d_{\alpha}, d_{2 n}, v_{2 n}\right)=(3,1.8,0.235)$, which is the minimum-energy AMD wave function with the assumption of a $2 \alpha$ core. The minimum-energy basis has an overlap of $\sim 82 \%$ with the state obtained by the full bases calculation (the largest overlaps with the ground state in the bases with $d_{\alpha}=2$ and $4 \mathrm{fm}$ are $\sim 67 \%$ and $\sim 61 \%$, respectively), and its binding energy is $55.80 \mathrm{MeV}$, which is $\sim 5 \mathrm{MeV}$ smaller than the ground-state binding energy in the full calculation. This indicates that the superposition of the basis wave functions significantly improves the ${ }^{10} \mathrm{Be}$ wave function. We plot in Fig. $3 f$ the absolute values of the $2 n$ overlap function $\left|f_{l=L=0}^{S=0}\right|$ projected onto the $r_{G}$ axis for the states obtained in the full

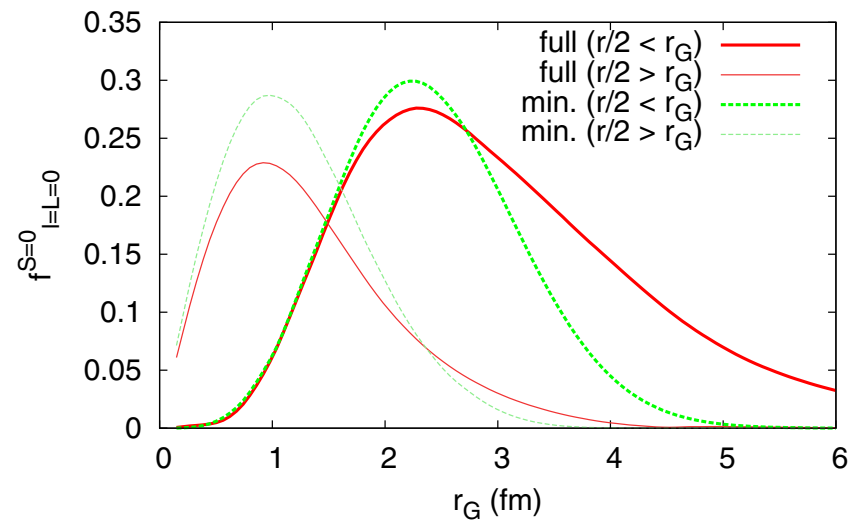

FIG. 3. The absolute values of the projection of the $2 n$ overlap functions of $f_{l=L=0}^{S=0}$ onto the $r_{G}$ axis in the full calculation and the calculation using the basis that gives the minimum energy. The red solid lines correspond to the full calculation and the green dashed lines are the calculation using only the minimum-energy basis. The thick lines are the dineutron component (the amplitudes in the $r / 2<r_{G}$ region) and the thin lines are the cigar component (the amplitudes in the $r / 2>r_{G}$ region). 
calculation and the minimum-energy basis calculation. In the calculation using the minimum-energy basis, the amplitudes of the dineutron $\left(r_{G} \sim 2 \mathrm{fm}\right)$ and cigar $\left(r_{G} \sim 1 \mathrm{fm}\right)$ peaks do not differ greatly, because the $0 p_{3 / 2}$ orbit (the lowest shell-model limit) is dominant for $2 n$ and the dineutron correlation mostly disappears in this basis.

A remarkable difference between these calculations is seen in the dineutron-tail component $\left(r_{G} \gtrsim 4 \mathrm{fm}\right)$. The minimum-energy basis does not have the dineutron tail expanded into the farther region because in a single basis, this tail has a rapidly dumping Gaussian form. However, in the full calculation where many bases with various $d_{2 n}$ and $v_{2 n}$ values are superposed, the dineutron tail is improved remarkably. This indicates that the present framework well describes the dineutron tail. It is also expected that this method can be useful for extremely loosely bound nuclei with neutron-halo or -skin structures. Further application to loosely bound nuclei is future work.

\section{SUMMARY}

In this study, we extended the AMD framework to describe dineutron correlation around a core with various structures. We first prepared the core wave functions using the cluster wave function or the AMD wave function, which are useful for describing various structure changes such as deformation and clustering. We described the detailed two-neutron motion around the cores considering the relative distance between the core and the two neutrons as the degree of freedom, and we superposed the basis wave functions with various core- $2 n$ distances to describe the competition between the dineutron formation and the $L S$ dissociation at the nuclear surface as well as the dineutron expansion far from the core. We additionally changed the Gaussian widths of the two neutrons to take into account the dineutron dispersion effect. Moreover, we constructed a $2 n$ overlap function as the analyzing method for investigating dineutron correlation around a core in detail. This method enabled us to visualize the spatial correlation between two neutrons and made the discussion clear.

As a first step, we applied the framework to the ground state of ${ }^{10} \mathrm{Be}$ and focused on the effect of the ${ }^{8} \mathrm{Be}$ core structure change on the dineutron correlation. In the present work, we assumed a $2 \alpha$ cluster structure for the ${ }^{8} \mathrm{Be}$ core and considered the fluctuation in the $\alpha-\alpha$ distance as the core structure change. Dineutron correlation was seen in the degree of mixing of different-parity single-particle orbits, i.e., the mixing of the minor $(s d)^{2}$ component into the major $p^{2}$ component for two neutrons in the ${ }^{10} \mathrm{Be}$ case. When the core was less deformed, the two neutrons were distributed near the core and greatly dissociated to the independent $\left(0 p_{3 / 2}\right)^{2}$ component due to the spin-orbit potential from the core; as a result, dineutron correlation was suppressed. However, when the core was well deformed by taking into account the fluctuation in the distance between $2 \alpha \mathrm{s}$, two neutrons could be expanded radially and they were favored to form a dineutron. Moreover, at the region far from the core, the spin-orbit potential becomes weaker and the $L S$ dissociation effect was suppressed. As a result, the dineutron correlation was enhanced at the surface and further regions due to the core structure change.

In this work, we applied the extended methods to ${ }^{10} \mathrm{Be}$ and showed that the present framework is useful to describe the dineutron component around the well-deformed core. Additionally, we concluded that the core structure significantly affects the dineutron correlation. Certainly, ${ }^{10} \mathrm{Be}$ is not a very loosely bound nucleus and the $L S$ dissociation effect on the dineutron plays an important role. However, we could see the asymmetry between the dineutron and cigar components indicating that the dineutron correlation somewhat occurs even though two neutrons are rather deeply bound. It is expected that dineutron correlation might be more or less apparent in other neutron-rich nuclei, even in those without an outstanding neutron-halo or -skin structure. In the future, we will apply the present framework to various nuclei and clarify the universal properties of dineutron correlation, e.g., the effect of the core excitation and clustering in addition to that of the core deformation on dineutron correlation and the effect of the $L S$ dissociation competing with the loose binding for two valence neutrons in a halo nucleus, through the systematic investigation.

\section{ACKNOWLEDGMENTS}

This work was supported by a Grant-in-Aid for Scientific Research from the Japan Society for the Promotion of Science (JSPS). A part of the computational calculations of this work was performed by using the supercomputers at YITP.

\section{APPENDIX: OVERLAP FUNCTIONS $\boldsymbol{f}$ AND $\boldsymbol{f}^{( \pm \pm)}$}

Here we show the details of the overlap functions $f$ and $f^{( \pm \pm)}$. We define the general $2 N$ overlap function $f$ as below:

$$
\begin{aligned}
f\left(\boldsymbol{r}, \boldsymbol{r}_{G}\right) \equiv & \mathcal{A}\left\langle\Phi_{\text {core }} \delta\left(\boldsymbol{r}_{A-1}-\boldsymbol{r}_{N_{1}}\right) \chi_{A-1} \tau_{A-1}\right. \\
& \times \delta\left(\boldsymbol{r}_{A}-\boldsymbol{r}_{N_{2}}\right) \chi_{A} \tau_{A}\left|\Phi_{\text {total }}\right\rangle, \\
\boldsymbol{r}= & \boldsymbol{r}_{N_{2}}-\boldsymbol{r}_{N_{1}}, \quad \boldsymbol{r}_{G}=\left(\boldsymbol{r}_{N_{1}}+\boldsymbol{r}_{N_{2}}\right) / 2,
\end{aligned}
$$

where $\Phi_{\text {total }}$ is the total wave function with $A$ nucleons whose center of mass of the core component is located at the origin and $\Phi_{\text {core }}$ is the core wave function with $A-2$ nucleons whose center of mass is located at the origin. $\chi_{A-1, A}$ and $\tau_{A-1, A}$ are the spin and isospin wave functions of the $(A-1)$ th or $A$ th nucleon and we can choose $\uparrow$ or $\downarrow$ for the spin components and $p$ or $n$ for the isospin components for each purpose. In the present work, we want to investigate the dineutron motion that is a spin-singlet pair of two neutrons so that $\chi_{A-1}=\uparrow$ and $\chi_{A}=\downarrow$, and $\tau_{A-1, A}=n$. In this Appendix, we show only this case but generalization is simple.

In the case of a spin-singlet $2 n$ pair, Eq. (A1), can be rewritten as

$$
f\left(\boldsymbol{r}, \boldsymbol{r}_{G}\right)=\sum_{i, j \in n}\left(\frac{4 v_{i} v_{j}}{\pi^{2}}\right)^{3 / 4} \exp \left[-v_{i}\left(\boldsymbol{r}_{n_{1}}-\boldsymbol{Y}_{i}\right)^{2}-v_{j}\left(\boldsymbol{r}_{n_{2}}-\boldsymbol{Y}_{j}\right)^{2}\right]\left\langle\chi_{\uparrow} \mid \chi_{i}\right\rangle\left\langle\chi_{\downarrow} \mid \chi_{j}\right\rangle \times \operatorname{det} B^{(i, j)}
$$




$$
\begin{aligned}
= & \sum_{i, j \in n}\left(\frac{4 v_{i} v_{j}}{\pi^{2}}\right)^{3 / 4} \exp \left[-\left(v_{i}+v_{j}\right)\left(r_{G}^{2}+r^{2} / 4\right)-v_{i} Y_{i}^{2}-v_{j} Y_{j}^{2}-\left(v_{i}-v_{j}\right) \boldsymbol{r} \cdot \boldsymbol{r}_{G} / 2\right] 4 \pi \sum_{l} \sum_{m} j_{l}\left(-i\left(v_{i} Y_{i}-v_{j} Y_{j}\right) r\right) \\
& \times Y_{l m}(\hat{\boldsymbol{r}}) Y_{l m}^{*}\left(\hat{\boldsymbol{Y}}_{r}\right) 4 \pi \sum_{l} \sum_{m} j_{l}\left(-2 i\left(v_{i} Y_{i}+v_{j} Y_{j}\right) r_{G}\right) Y_{l m}\left(\hat{\boldsymbol{r}}_{G}\right) Y_{l m}^{*}\left(\hat{\boldsymbol{Y}}_{G}\right)\left\langle\chi_{\uparrow} \mid \chi_{i}\right\rangle\left\langle\chi_{\downarrow} \mid \chi_{j}\right\rangle \times \operatorname{det} B^{(i, j)},
\end{aligned}
$$

where $j_{l}$ are the spherical Bessel functions and $Y_{l m}$ are the spherical harmonics. $\hat{\boldsymbol{Y}}_{r, G}$ are the polar angles of the vectors $v_{i} \boldsymbol{Y}_{i}-v_{j} \boldsymbol{Y}_{j}$ or $v_{i} \boldsymbol{Y}_{i}+v_{j} \boldsymbol{Y}_{j}$, respectively. $B^{(i, j)}$ is an $(A-2) \times(A-2)$ norm matrix composed of $\left\langle\varphi_{\text {core }, \kappa}\right|(\kappa=$ $1, \ldots, A-2)$ in $\left\langle\Phi_{\text {core }}\right|$ and $\left|\varphi_{\text {total }, \kappa}\right\rangle(\kappa=1, \ldots, A)$ in $\left|\Phi_{\text {total }}\right\rangle$ except for the $i$ th and $j$ th single-particle wave functions. $f\left(\boldsymbol{r}, \boldsymbol{r}_{G}\right)$ is totally antisymmetrized by $\mathcal{A}$ and hence $B^{(j, i)}=-B^{(i, j)}$. We perform the angular integrals $\int d^{2} \hat{\boldsymbol{r}} Y_{00}(\hat{\boldsymbol{r}}) \int d^{2} \hat{\boldsymbol{r}}_{G} Y_{00}\left(\hat{\boldsymbol{r}}_{G}\right)$ to project the relative and centerof-mass motions of $2 n$ onto $l=L=0$, and we additionally neglect the term proportional to $\boldsymbol{r} \cdot \boldsymbol{r}_{G}$ in the exponential term in Eq. (A4), resulting in $f_{l=L=0}^{S=0}\left(r, r_{G}\right)$ as

$$
\begin{aligned}
f_{l=L=0}^{S=0}\left(r, r_{G}\right)= & \sum_{i, j \in n}\left(\frac{4 v_{i} v_{j}}{\pi^{2}}\right)^{3 / 4} \exp \left[-\left(v_{i}+v_{j}\right)\left(r_{G}^{2}+r^{2} / 4\right)\right. \\
& \left.-v_{i} Y_{i}^{2}-v_{j} Y_{j}^{2}\right] 4 \pi j_{0}\left(-i\left(v_{i} Y_{i}-v_{j} Y_{j}\right) r\right) \\
& \times j_{0}\left(-2 i\left(v_{i} Y_{i}+v_{j} Y_{j}\right) r_{G}\right) \\
& \times\left\langle\chi_{\uparrow} \mid \chi_{i}\right\rangle\left\langle\chi_{\downarrow} \mid \chi_{j}\right\rangle \times \operatorname{det} B^{(i, j)} .
\end{aligned}
$$

This $2 n$ overlap function only depends on the absolute values of $r=|\boldsymbol{r}|$ and $r_{G}=\boldsymbol{r}_{G}$. We calculate the probability of the spin-singlet $2 n$ pair, $P_{l=L=0}^{S=0}$, the root-mean-square distance between two neutrons $\sqrt{\left\langle r^{2}\right\rangle_{l=L=0}^{S=0}}$, and that between the two neutrons and the core $\sqrt{\left\langle r_{G}^{2}\right\rangle_{l=L=0}^{S=0}}$ simply using the numerical integrals of $r$ and $r_{G}$ [Eqs. (13), (14), and (15)].

We calculate the $(++)$ and $(--)$ components $f_{l=L=0}^{S=0( \pm \pm)}$, from the $2 n$ overlap function $f_{l=L=0}^{S=0}$ as Eq. (18). Here, we show that $f_{l=L=0}^{S=0( \pm \pm)}$, defined in Eq. (18), is certainly equivalent to the components where both neutrons are projected to positive- or negative-parity single-particle orbits if the relative and centerof-mass motions of $2 n$ are projected as $l=L=0$. We begin by projecting both neutrons in the $2 n$ overlap function $f\left(\boldsymbol{r}, \boldsymbol{r}_{G}\right)$ to the positive- or negative-parity single-particle orbits. Noting the definition of the coordinates $\boldsymbol{r}$ and $\boldsymbol{r}_{G}$ in Eq. (A2), we have

$$
\begin{aligned}
(1 \pm & \left.\mathcal{P}_{\boldsymbol{r}_{n 1}}\right) / 2 \times\left(1 \pm \mathcal{P}_{\boldsymbol{r}_{n 2}}\right) / 2 \times f\left(\boldsymbol{r}, \boldsymbol{r}_{G}\right) \\
= & \frac{1}{4}\left[\left(1+\mathcal{P}_{\boldsymbol{r}_{n 1}} \mathcal{P}_{\boldsymbol{r}_{n 2}}\right) \pm\left(\mathcal{P}_{\boldsymbol{r}_{n 1}}+\mathcal{P}_{\boldsymbol{r}_{n 2}}\right)\right] f\left(\boldsymbol{r}, \boldsymbol{r}_{G}\right) \\
= & \frac{1}{4}\left[\left(f\left(\boldsymbol{r}, \boldsymbol{r}_{G}\right)+f\left(-\boldsymbol{r},-\boldsymbol{r}_{G}\right)\right) \pm\left(f\left(2 \boldsymbol{r}_{G}, \boldsymbol{r} / 2\right)\right.\right. \\
& \left.\left.+f\left(-2 \boldsymbol{r}_{G},-\boldsymbol{r} / 2\right)\right)\right] .
\end{aligned}
$$

If we project $f$ to $l=L=0$ with the operator $\mathcal{P}_{l=L=0}$, $f_{l=L=0}^{S=0}$ becomes just a function of the absolute values of $r$ and $r_{G}\left[\mathcal{P}_{l=L=0} f\left(\boldsymbol{r}, \boldsymbol{r}_{G}\right)=f_{l=L=0}^{S=0}\left(r, r_{G}\right)\right]$. As a result of the projection to $l=L=0$, the former two terms and the latter two terms on the last line of Eq. (A6) give the same contributions, respectively. We can therefore rewrite Eq. (A6) under the projection to $l=L=0$ as

$$
\begin{aligned}
\mathcal{P}_{l}=L=0 & {\left[\left(1 \pm \mathcal{P}_{\boldsymbol{r}_{n 1}}\right) / 2 \times\left(1 \pm \mathcal{P}_{\boldsymbol{r}_{n 2}}\right) / 2 \times f\left(\boldsymbol{r}, \boldsymbol{r}_{G}\right)\right] } \\
& =\frac{1}{2}\left(f_{l=L=0}^{S=0}\left(r, r_{G}\right) \pm f_{l=L=0}^{S=0}\left(2 r_{G}, r / 2\right)\right) \\
& =f_{l=L=0}^{S=0( \pm \pm)} .
\end{aligned}
$$

This means that $f_{l=L=0}^{S=0( \pm \pm)}$ defined in Eq. (18) are certainly the components in the $2 n$ overlap function where both neutrons are projected to the positive- or negative-parity single-particle orbits if the relative and center-of-mass motions of the two neutrons are projected as $l=L=0$.
[1] M. Baldo, J. Cugnon, A. Lejeune, and U. Lombardo, Nucl. Phys. A 515, 409 (1990).

[2] M. Matsuo, Phys. Rev. C 73, 044309 (2006).

[3] G. F. Bertsch and H. Esbensen, Ann. Phys. (N.Y.) 209, 327 (1991).

[4] M. V. Zhukov, B. V. Danilin, D. V. Fedrov, J. M. Bang, I. J. Thompson, and J. S. Vaagen, Phys. Rep. 231, 151 (1993).

[5] K. Arai, Y. Ogawa, Y. Suzuki, and K. Varga, Prog. Theor. Phys. Suppl. 142, 97 (2001).

[6] P. Descouvemont, C. Daniel, and D. Baye, Phys. Rev. C 67, 044309 (2003).

[7] M. Matsuo, K. Mizuyama, and Y. Serizawa, Phys. Rev. C 71, 064326 (2005).

[8] K. Hagino and H. Sagawa, Phys. Rev. C 72, 044321 (2005).

[9] P. Descouvemont, E. Tursunov, and D. Baye, Nucl. Phys. A 765, 370 (2006).
[10] Y. Kanada-En'yo, Phys. Rev. C 76, 044323 (2007).

[11] K. Hagino, H. Sagawa, J. Carbonell, and P. Schuck, Phys. Rev. Lett. 99, 022506 (2007).

[12] N. Pillet, N. Sandulescu, and P. Schuck, Phys. Rev. C 76, 024310 (2007).

[13] N. Itagaki, M. Ito, K. Arai, S. Aoyama, and T. Kokalova, Phys. Rev. C 78, 017306 (2008).

[14] N. Pillet, N. Sandulescu, P. Schuck, and J.-F. Berger, Phys. Rev. C 81, 034307 (2010).

[15] F. Kobayashi and Y. Kanada-En'yo, Phys. Rev. C 86, 064303 (2012).

[16] F. Kobayashi and Y. Kanada-En'yo, Phys. Rev. C 88, 034321 (2013).

[17] F. Kobayashi and Y. Kanada-En'yo, Phys. Rev. C 89, 024315 (2014).

[18] L. V. Grigorenko, R. C. Johnson, I. G. Mukha, I. J. Thompson, and M. V. Zhukov, Phys. Rev. Lett. 85, 22 (2000). 
[19] L. V. Grigorenko, R. C. Johnson, I. G. Mukha, I. J. Thompson, and M. V. Zhukov, Phys. Rev. C 64, 054002 (2001).

[20] L. V. Grigorenko and M. V. Zhukov, Phys. Rev. C 68, 054005 (2003).

[21] L. V. Grigorenko and M. V. Zhukov, Phys. Rev. C 76, 014008 (2007).

[22] L. V. Grigorenko, T. D. Wiser, K. Miernik, R. J. Charity, M. Pfützner, A. Banu, C. R. Bingham, M. Ćwiok, I. G. Darby, W. Dominik et al., Phys. Lett. B 677, 30 (2009).

[23] L. V. Grigorenko, T. D. Wiser, K. Mercurio, R. J. Charity, R. Shane, L. G. Sobotka, J. M. Elson, A. H. Wuosmaa, A. Banu, M. McCleskey et al., Phys. Rev. C 80, 034602 (2009).

[24] H. T. Johansson, Y. Aksyutina, T. Aumann, K. Boretzky, M. J. G. Borge, A. Chatillon, L. V. Chulkov, D. Cortina-Gil, U. D. Pramanik, H. Emling et al., Nucl. Phys. A 847, 66 (2010).

[25] A. Spyrou, Z. Kohley, T. Baumann, D. Bazin, B. A. Brown, G. Christian, P. A. DeYoung, J. E. Finck, N. Frank, E. Lunderberg et al., Phys. Rev. Lett. 108, 102501 (2012).

[26] I. A. Egorova, R. J. Charity, L. V. Grigorenko, Z. Chajecki, D. Coupland, J. M. Elson, T. K. Ghosh, M. E. Howard, H. Iwasaki, M. Kilburn et al., Phys. Rev. Lett. 109, 202502 (2012).

[27] L. V. Grigorenko, I. A. Egorova, R. J. Charity, and M. V. Zhukov, Phys. Rev. C 86, 061602(R) (2012).

[28] Z. Kohley, E. Lunderberg, P. A. DeYoung, A. Volya, T. Baumann, D. Bazin, G. Christian, N. L. Cooper, N. Frank, A. Gade et al., Phys. Rev. C 87, 011304(R) (2013).

[29] Z. Kohley, T. Baumann, G. Christian, P. A. DeYoung, J. E. Finck, N. Frank, B. Luther, E. Lunderberg, M. Jones, S. Mosby et al., Phys. Rev. C 91, 034323 (2015).
[30] Y. Kanada-En'yo and F. Kobayashi, Phys. Rev. C 90, 054332 (2014).

[31] Y. Kanada-En'yo, H. Morita, and F. Kobayashi, Phys. Rev. C 91, 054323 (2015).

[32] Y. Kanada-En'yo, H. Horiuchi, and A. Ono, Phys. Rev. C 52, 628 (1995).

[33] Y. Kanada-En'yo and H. Horiuchi, Phys. Rev. C 52, 647 (1995).

[34] Y. Kanada-En'yo and H. Horiuchi, Prog. Theor. Phys. Suppl. 142, 205 (2001).

[35] N. Furutachi, M. Kimura, A. Doté, and Y. Kanada-En'yo, Prog. Theor. Phys. 122, 865 (2009).

[36] H. Feldmeier, K. Bieler, and J. Schnack, Nucl. Phys. A 586, 493 (1955).

[37] T. Neff and H. Feldmeier, Nucl. Phys. A 713, 311 (2003).

[38] Y. Taniguchi, M. Kimura, and H. Horiuchi, Prog. Theor. Phys. 112, 475 (2004).

[39] T. Suhara and Y. Kanada-En'yo, Prog. Theor. Phys. 123, 303 (2010).

[40] F. Kobayashi and Y. Kanada-En'yo, Prog. Theor. Phys. 126, 457 (2011).

[41] A. Volkov, Nucl. Phys. 74, 33 (1965).

[42] R. Tamagaki, Prog. Theor. Phys. 39, 91 (1968).

[43] A. Ozawa, T. Suzuki, and I. Tanihata, Nucl. Phys. A 693, 32 (2001).

[44] W. Nörtershäuser, D. Tiedemann, M. Žáková, Z. Andjelkovic, K. Blaum, M. L. Bissell, R. Cazan, G. W. F. Drake, C. Geppert, M. Kowalska et al., Phys. Rev. Lett. 102, 062503 (2009).

[45] I. Angeli, At. Data Nucl. Data Tables 87, 185 (2004).

[46] J. L. Friar, J. Martorell, and D. W. L. Sprung, Phys. Rev. A 56, 4579 (1997). 